\title{
Uncovering the Distribution of Option Implied Risk Aversion
}

\author{
Maria Kyriacou$^{1}$, Jose Olmo $^{2 *}$ (D), Marius Strittmatter ${ }^{1}$ \\ ${ }^{1}$ Economics Department, University of Southampton, Southampton, UK \\ ${ }^{2}$ Department of Economic Analysis, Universidad de Zaragoza, Zaragoza, Spain \\ Email: M.Kyriacou@soton.ac.uk, *joseolmo@unizar.es,ms2g15@soton.a c.uk
}

How to cite this paper: Kyriacou, M., Olmo, J. and Strittmatter, M. (2019) Uncovering the Distribution of Option Implied Risk Aversion. Journal of Mathematical Finance, 9, 81-104. https://doi.org/10.4236/jmf.2019.92006

Received: January 30, 2019

Accepted: March 11, 2019

Published: March 14, 2019

Copyright () 2019 by author(s) and Scientific Research Publishing Inc. This work is licensed under the Creative Commons Attribution International License (CC BY 4.0).

http://creativecommons.org/licenses/by/4.0/

\section{(c) (i) Open Access}

\begin{abstract}
This paper explores the dynamics of risk aversion of a representative agent with an iso-elastic utility function. In contrast to most of the existing literature, we estimate the coefficient of relative risk aversion from option prices. To do this, we transform the risk-neutral density function obtained from a cross-section of option prices to an objective distribution function that reflects individuals' risk aversion through a CRRA utility function. The dynamics of the relative risk-aversion coefficient are obtained by repeating the same estimation procedure over rolling windows. This procedure uncovers strong variation in risk aversion over time. We also propose a simulation procedure to construct confidence intervals for the risk-aversion coefficient in each period. We assess the robustness of these confidence intervals under different assumptions on the data generating process of stock prices. The results imply a strong influence of volatility on the variation of risk aversion. In an empirical application, we compare the forecasting performance of our approach based on our risk-aversion estimates against the method proposed in [1]. Overall, we find that our simulation based approach obtains better forecasting results than bootstrap methods.
\end{abstract}

\section{Keywords}

Simulation Based Risk-Aversion, Empirical Pricing Kernel, Index Options, Risk-Transformations

\section{Introduction}

Stock options are priced using risk-neutral expectations of the payoff of the underlying asset. These risk-neutral expectations usually incorporate in the option pricing formula a flexible specification of the density function for 
describing the price of the underlying asset. Stock prices are priced, instead, by discounting the expected value of the future cash flows of the asset under an objective distribution function. Both approaches incorporate individuals' attitude towards risk. The risk-neutral approach embeds individuals' risk aversion in the risk-neutral probability distribution of the risky payoffs, that are discounted using the risk-free rate. In contrast, the objective approach uses objective probabilities, inferred from historical prices or alternative parametric methods, and discount future payoffs using a stochastic discount factor that incorporates a risk premium over the risk-free rate.

There are different approaches to connect the risk-neutral valuation with the objective valuation. A convenient formulation that relies on an utilitarian approach is to assume a parametric relationship between the distribution functions through a utility function. The analytical tractability of power utility functions offers a convenient modelling device for describing individuals' preferences. In this paper we exploit this parametric relationship between the risk-neutral and objective distribution functions and the power utility function to extract consistent estimates of the coefficient of relative risk aversion of a representative agent. There are two reasons for extracting risk aversion from risk-neutral valuations of option prices. First, risk-aversion estimates are economically important and provide relevant information for understanding the performance and dynamics of financial markets. Second, the empirical finance literature confirms the significant improvement in the ability of parametric models for forecasting stock prices that incorporate reliable estimates of risk aversion.

The link between expected returns and investors' risk-preferences in financial markets is the foundation of research concerning the risk-return relationship. Several methods have been developed over time in order to determine the risk-premia underlying asset markets. Popular approaches are, for example, the standard and consumption-based capital asset pricing model. These pioneering asset pricing methods are usually estimated based on asset price returns, treasury yields and consumption data. Although these models are still the building blocks of current asset pricing models and the foundation of today's financial economics, their success in estimating the underlying risk premia in financial markets has been limited. A prominent example is the seminal article by [2] on the equity premium puzzle. These authors retrieve extremely high values of the risk aversion coefficient in order for the asset pricing model to be compatible with observed asset prices. These values are not economically justified. Other studies have tried to correct these estimates by imposing different assumptions on individuals' preferences. For example, [3] makes strong assumptions on the flexibility of the underlying utility function. This author finds estimates of the risk aversion coefficient equal to one and consistent with the presence of a log-utility function for modelling the preferences of a representative agent. [4] find values of the risk aversion coefficient in the range of two. [5] in their 
seminal study propose a novel GMM econometric framework for estimating the model parameters, however, their parameter estimates are consistent with previous estimates and oscillate between 0 and 1 [2], discussed above, find values of the risk aversion coefficient around 55 that are difficult to reconcile with practitioners' and financial economists' expectations on the underlying risk aversoin in financial markets. [6] propose a recursive utility function for modelling individuals' preferences and find under this novel approach a risk aversion coefficient that oscillates between 0.4 and 1.4. It is important to remark that the Epstein-Zin utility function is able to disentangle individuals' risk aversion from the rate of intertemporal substitution. [7] propose habit formation models in which individuals' preferences depend on a reference point under which perceived utility of the asset is zero. This approach provides more variability in the estimates of the risk aversion coefficient. Thus, these authors find a risk aversion coefficient between 0 and 12. [8] $\mathrm{n}$ an asset pricing context find values in the range of 40 - 50 consistent with those found in [2]. [9] and [10] find values between 5.4 and 11.9 and smaller than 3, respectively. More recently, [11] derives a coefficient of risk aversion of 12.7 and [12] using option prices close to their asset price returns and consumption data find a value of the relative risk aversion coefficient equal to 3.52. These authors exploit option prices to extract implied volatility measures for estimating the risk aversion coefficient.

The conclusion of this literature on risk aversion is that although it well acknowledges the existence of a premium in financial markets to compensate individuals for the presence of risk in asset prices under individuals' risk aversion, there is much controversy on how to measure it and what values are consistent with the realizations of stock prices. There are three major approaches for retrieving risk-aversion coefficients based on option prices. They all try to combine risk-neutral dynamics from option prices with the realized dynamics of asset prices. The first approach estimates the risk-neutral density based on option prices separately from the density of asset prices obtained from historical asset price data. Afterwards, both densities are compared against each other to retrieve the risk preferences of market participants [11] [13]-[20]. The second approach jointly estimates the continuous-time dynamics of the risk-neutral and the subjective process. Option and asset prices are used to jointly estimate the pricing kernel that incorporates the premia for price, volatility and jump risks. [21] [22] [23] [24]. The third approach assumes a parametric form of the riskaversion function, which is combined with the risk-neutral density in order to derive the subjective density [1] [19] [24] [25] [26] [27].

Our aim is to follow the third approach, and more specifically, [1]. One of our objectives is to gain insight on the variation of risk-aversion estimates over time and, hence, through the business cycle. [1] provide quantitative methods to evaluate risk-aversion estimates. These authors introduce two different approaches. First, a Monte-Carlo simulation method to retrieve the standard error 
of risk-aversion estimates. Second, a Bootstrapping method to capture the influence of actual data and potential model misspecification on the reliability of risk-aversion estimates. Despite the appealing of both approaches, these methods face challenges due to the limited availability of asset prices and option prices. To overcome this issue, we explore different parametric data generating processes for constructing the risk-neutral distribution function for describing the dynamics of option and asset prices. Variation in the estimates of risk aversion is obtained by constructing rolling windows of 120 monthly observations and obtaining estimates of risk aversion for each window. This number is sufficiently large to avoid too much variation in risk-aversion estimates at the same time small enough to capture changes in market conditions that trigger shifts in risk aversion. Confidence intervals of the risk-aversion estimates can be constructed by simulating the prices from a parametric data generating process. The simulated distribution of risk-aversion estimates provides valuable insights on the variation of the risk-aversion coefficient. Furthermore, in the proposed set-up the underlying asset price process allows for different characteristics starting with the base case of a Geometric Brownian Motion and its extension to accommodate stochastic volatility processes. The simulation results are compared with the classic approach followed by [1] nd [27] These authors derive their risk-aversion estimates based on past series of risk-neutral densities and realised asset prices.

Our extensive simulation exercise provides the following insights. First, our results suggest that there is more variation in the risk-aversion coefficient than implied by [1]. Not surprisingly, the volatility component has a major impact on the coefficient of relative risk aversion. The choice of parametric model for describing asset prices also has a major effect on the estimates of risk aversion for a representative agent. Thus, we note that imposing a lognormal density on asset prices produces greater variation in the coefficient of risk aversion compared to the mixture-lognormal and generalised beta distribution of the second kind. Lastly, we also perform a forecasting exercise to assess the relative out-of-sample ability of the different data generating processes for estimating the risk-aversion coefficient for forecasting stock prices. The forecasting performance is evaluated using the subjective density derived from the average simulated risk-aversion estimate. The findings are compared against the classic approach obtained from [1]. Overall, we find that the simulation based approach obtains better forecasting results than bootstrap methods. This fact is particularly apparent in the period after the sub-prime crisis.

The paper is structured in six sections. Section 2 introduces the considered density types and transformation from risk-neutral to subjective density. Section 3 describes the proposed simulation method. Section 4 elaborates on the simulation results. Section 5 provides the summary statistics of our observed S\&P 500 option and index prices. Further, estimation errors and risk-neutral characteristics are reported, and empirical results are discussed. Lastly, Section 6 
concludes the main findings and gives suggestions for further research.

\section{Option Implied Densities}

The risk-aversion estimates obtained in this section are retrieved from option prices. This approach goes back to the density forecasting literature for option pricing as, for example, [27]. The foundations to this literature on option pricing is due to [28]. These authors show that when there are no-arbitrage opportunities and options are available for all strikes $K$, a call-option $c$ can be evaluated by:

$$
c(K)=\mathrm{e}^{-r T} \int_{K}^{\infty}(S-K) f_{Q}(S) \mathrm{d} S,
$$

where $S$ is the stock price, $r$ the risk-free rate, $T$ the time to maturity and $f_{Q}$ a unique risk-neutral density. All parameters can be observed in the market except for the risk-neutral density. Assuming a parametric form of $f_{Q}$, its parameters $\theta$ can be obtained by minimising the squared error between observed $c_{o}$ and model-based $c_{m}$ call prices:

$$
\underset{\theta}{\arg \min } G(\theta)=\sum_{i=1}^{N}\left(c_{o}\left(K_{i}\right)-c_{m}\left(K_{i} \mid \theta\right)\right)^{2},
$$

with $N$ the number of options in the cross-section. The risk-neutral valuation involving the density function $f_{Q}$ is restricted by the risk-neutrality constraint $F=E^{Q}\left[S_{T}\right]$, stating that the expected value of $f_{Q}$ equals the forward price $F$. To reflect the dynamics of the underlying asset, the risk-neutral density needs to be transformed into a subjective counterpart density function that incorporates individuals' risk-aversion profile. We follow the transformation in [1], who relate the risk-neutral $f_{Q}$ to a subjective density function $f_{P}$ by making an assumption about the preferences of a representative investor. This parametric relationship goes back to [11]. In our study, we assume a power utility function $u$ with $u^{\prime}=x^{-\gamma}$ :

$$
f_{P}\left(S_{T}\right)=\frac{\frac{f_{Q}\left(S_{T}\right)}{u^{\prime}\left(S_{T}\right)}}{\int_{0}^{\infty} \frac{f_{Q}(y)}{u^{\prime}(y)} \mathrm{d} y}=\frac{S_{T}^{-\gamma} f_{Q}\left(S_{T}\right)}{\int_{0}^{\infty} y^{-\gamma} f_{Q}(y) \mathrm{d} y} .
$$

The constant relative risk-aversion parameter $\gamma$, inherited from the power utility and responsible for the transformation into the subjective density, is the central aspect of the subsequent analysis. As in [27], its value is determined by a series of $M$ densities, maximising the log-likelihood of the realization of asset prices:

$$
\log \left(L\left(S_{T, 1}, S_{T, 2}, \cdots, S_{T, M} \mid \gamma\right)\right)=\sum_{i=1}^{M} \log \left(f_{P, i}\left(S_{T, i} \mid \hat{\theta}_{i}, \gamma\right)\right)
$$

In our empirical analysis, we consider three different density types with different degrees of flexibility: Lognormal density (LN), mixture-lognormal density (MLN) and generalized beta density of the second kind (GB2). Their 
characteristics and transformation are outlined in the subsequent subsections.

\subsection{Mixtures of Lognormal Densities}

[29] introduces a mixture of lognormal densities, MLN herafter, to evaluate option prices. Following [27], this distribution is defined as a weighted combination of two lognormal densities. A European call price is evaluated as the sum of two [30] models:

$$
c(K \mid \theta)=w c_{B}\left(F_{1}, T, K, r, \sigma_{1}\right)+(1-w) c_{B}\left(F_{2}, T, K, r, \sigma_{2}\right) .
$$

with $\theta=\left(F_{1}, \sigma_{1}, F_{2}, \sigma_{2}, w\right)$. The risk-neutrality constraint for the MLN reduces the amount of free parameters to four and by stating that:

$$
F=w F_{1}+(1-w) F_{2},
$$

with $F$ the forward price. Transforming the density into its subjective counterpart as in (3) leads to another mixture-lognormal density. The subjective parameters become:

$$
\begin{gathered}
\tilde{\theta}=\left(\tilde{F}_{1}, \tilde{F}_{2}, \sigma_{1}, \sigma_{2}, \tilde{w}\right), \\
\tilde{F}_{i}=F_{i} \mathrm{e}^{\gamma \sigma^{2} T}, \text { for } i=1,2 \text {, and } \\
\frac{1}{\tilde{w}}=1+\frac{1-w}{w}\left(\frac{F_{2}}{F_{1}}\right)^{\gamma} \exp \left(0.5\left(\gamma^{2}-\gamma\right)\left(\sigma_{2}^{2}-\sigma_{1}^{2}\right) T\right) .
\end{gathered}
$$

The lognormal density (LN) is a special case of the above for $w=1$. As a result, we do not describe this distribution separately.

\subsection{Generalized Beta Densities}

The generalized beta density of the second kind, GB2 hereafter, was introducted by [31] and has been applied to option prices by [27]. They show that a European call option price can be evaluated by:

$$
\begin{aligned}
c(K \mid \theta)= & F \mathrm{e}^{-r T}\left[1-F_{\beta}(v(K, a, b) \mid p+1 / a, q-1 / a)\right] \\
& -K \mathrm{e}^{-r T}\left[1-F_{\beta}(v(K, a, b) \mid p, q)\right],
\end{aligned}
$$

with $\theta=(a, b, p, q)$, all greater than zero, $v(K, a, b)=(x / b)^{a} /\left[1+(x / b)^{a}\right]$ and $F_{\beta}$ the distribution function of a random variable following a beta distribution. The risk-neutrality constraint reduces the amount of free parameters from four to three:

$$
F=\frac{b B(p+1 / a, q-1 / a)}{B(p, q)}, a q>1,
$$

where $B$ is defined in terms of gamma functions $B(p, q)=\Gamma(p) \Gamma(q) / \Gamma(p+q)$. The transformation of the GB2 risk-neutral density as in (3) leads a different type of GB2 density function. The subjective parameters are then $\tilde{\theta}=\left(a, b, p+\frac{\gamma}{a}, q-\frac{\gamma}{a}\right)$ with $a q>\gamma$.

\section{Risk Aversion Evaluation}

One of the biggest challenges in the evaluation of risk-aversion estimates is the 
limited availability of data. While option prices provide an accurate description of risk-neutral market expectations, subjective dynamics can only be observed as single price outcomes obtained from market prices. Linking a single risk-neutral density to realized asset prices to estimate the degree of risk aversion of a representative agent produces high variation in the parameter estimates. One way of overcoming this issue is to obtain risk aversion over several periods, as presented in (4). This approach requires, however, access to a sufficiently long dataset. In situations where the time horizon is too short, estimates might be misleading and will not accurately reflect the risk preferences of the representative agent.

[1] introduce a Monte-Carlo simulation method and a Bootstrapping procedure to gain better insight into the variation of risk-aversion estimates. However, their approach does not overcome the problem of single price realisations. More importantly, risk aversion is only estimated from past data, which may accurately reflect individuals' past preferences but not necessarily those relevant for the forthcoming period.

We propose an alternative Monte-Carlo simulation exercise that addresses all these problems. Our simulation setting is structured in a way that only considers the most recent risk-neutral dynamics and simulates potential asset price outcomes based on different stochastic processes. This allows us to estimate risk aversion by taking into account current market dynamics. Importantly, it is possible to simulate several asset price outcomes for the same time period providing a more detailed outlook of the anticipated variation of the underlying risk aversion.

\subsection{Simulation Framework}

In our simulation framework current market dynamics are captured by a set of assumptions on risk-neutral and subjective processes. Given these, we can simulate a whole range of potential asset price outcomes for a specific period and use these to estimate risk aversion. Simulation-based risk-aversion estimates provide better insight into the full spectrum of potential risk-aversion estimates. Accordingly, the utility function can be validated. The Monte-Carlo simulation framework consists of the following five steps ${ }^{1}$ :

1) Make assumptions about the subjective process that includes the utility function of the representative agent and the risk-neutral process.

2) Obtain options from the risk-neutral process and fit a risk-neutral density into option prices. Since, the risk-neutral process does not change, estimated risk-neutral parameter values do not change for the time horizon of $M$ periods.

3) Generate $M$ asset price outcomes based on the subjective process for $M$ risk-neutral densities.

4) Estimate the risk-aversion parameter $\gamma$ over the estimation horizon of $M$ periods based on (4).

${ }^{1}$ Note that for the empirical application, Step 2 does not draw option prices from the risk-neutral process since options can be observed directly in the market. 
5) Repeat Steps 3 and $4 H$ times.

\subsection{Likelihood Criteria}

Log-likelihood based tests on the asset price outcomes can be useful in providing insights on the quality of the retrieved risk-aversion values, as used in the work of [32], [27] and [24] to compare the quality of their density methods on equity indices. The log-likelihood function is defined as:

$$
\log L_{m}=\sum_{t=1}^{i} \log \left(f_{m, t}\left(S_{t+1}\right)\right)
$$

where $f$ is the density function, $m$ the density type and $i$ the number of densities. The log-likelihood function serves two different testing purposes. First, we construct a log-likelihood ratio test to assess statistically whether the additional risk-aversion parameter $\gamma$ significantly improves the forecasting performance of the densities.

$$
\begin{aligned}
& H_{0}: \gamma=0 \\
& H_{1}: \gamma \neq 0 .
\end{aligned}
$$

The corresponding test statistic is defined as:

$$
2^{*} L R=-2\left(L_{m, \gamma=0}-L_{m, \gamma \neq 0}\right) \text {. }
$$

Under the null $2^{*} L R \sim \chi^{2}$ distribution with 1 degree of freedom.

Second, we test whether the two competing methods, denoted as $m$ and $n$, provide equal expected log-likelihoods as described:

$$
\begin{aligned}
& H_{0}: E\left[\log \left(f_{m, t}\left(S_{t+1}\right)\right)\right]=E\left[\log \left(f_{n, t}\left(S_{t+1}\right)\right)\right] \\
& H_{1}: E\left[\log \left(f_{m, t}\left(S_{t+1}\right)\right)\right] \neq E\left[\log \left(f_{n, t}\left(S_{t+1}\right)\right)\right]
\end{aligned}
$$

This test is proposed by [33], AG hereafter, and has been applied to option based densities by [24]. The AG test is an out-of sample test, which uses a rolling window estimation scheme to rank density forecasts. Its loss function is based on a weighted Likelihood ratio test and offers the advantage that it can be robust to heterogeneous data sets, while it can be applied to densities obtained from both parametric and non-parametric models. The test is based on the difference of log-likelihood of each period, denoted by $d_{t}$. The test statistic, AG, is retrieved on the basis of the average difference $\hat{d}$ and standard deviation of $s_{d}$ of $d$ with integer times $i, \cdots, j$, as:

$$
A G=(j-i+1)^{0.5} \frac{\hat{d}}{s_{d}} .
$$

Under the null hypothesis $H_{0}$, it follows that $A G \sim N(0,1)$.

\subsection{Market Assumptions and Estimation}

We impose two different parametric forms to model market dynamics. First, market dynamics are assumed to follow a Geometric Brownian Motion as in [34]. Second, we incorporate stochastic volatility into the model by implementing 
the model by [35]. In both cases, we assume that the volatility parameters are derived from option prices including also the subjective dynamics. Only the drift parameter is changed between specifications in order to account for the riskpremium of the subjective approach. This transformation is similar to the risk-premium transformation in [24]. The drift term of the subjective process is estimated using historical asset price returns under the assumption of lognormality of stock prices. Finally, we illustrate how expected risk aversion can be obtained by taking into account the risk-neutral and subjective dynamics.

\subsubsection{Geometric Brownian Motion}

In the first specification, we assume that markets follow a Geometric Brownian Motion as in [34]:

$$
\mathrm{d} S_{t}=\mu S_{t} \mathrm{~d} t+\sigma S_{t} \mathrm{~d} W_{t} \quad \text { with } W_{t} \sim \mathcal{N}(0,1)
$$

with $\mu$ the drift term and $\sigma$ the constant volatility. Option prices can be evaluated by the well-known Black and Scholes formula as follows.

$$
\begin{gathered}
c(X)=S_{t} \mathrm{e}^{-q T} N\left(d_{1}\right)-X \mathrm{e}^{-r T}\left(d_{2}\right) \\
d_{1}=\frac{\ln \left(\frac{S_{t}}{X}\right)+\left(r-q+\frac{\sigma^{2}}{2}\right) T}{\sigma \sqrt{T}} \\
d_{2}=d_{1}-\sigma \sqrt{T} .
\end{gathered}
$$

The parameter estimation is performed as in (2) by only considering a single at-the-money option.

\subsubsection{Stochastic Volatility}

An alternative to the geometric Brownian motion is the stochastic volatility model of [35]. This specification allows for more flexibility and captures more accurately observed market dynamics. As in the previous model, the change in asset prices follows a Geometric Brownian Motion as in (17), whereas the volatility parameter $\sigma$ follows an Ornstein-Uhlenbeck process.

$$
\mathrm{d} \sigma_{t}=\kappa\left(\theta-v_{t}\right) \mathrm{d} t+\xi \sqrt{v_{t}} \mathrm{~d} Z_{t} \quad \text { with } Z_{t} \sim \mathcal{N}(0,1)
$$

where $\mu$ denotes the drift term, $\sigma$ the volatility of stock price, $\kappa$ the mean reversion speed, $\theta$ the long-run variance, $v_{t}$ the current variance and $\xi$ the volatility of volatility parameter. [35] provides a closed form solution to price a European call option in the form of the Black and Scholes formula:

$$
c(X)=S \mathrm{e}^{-q T} P_{1}-X \mathrm{e}^{-r T} P_{2}
$$

In contrast to the estimation of the risk-neutral density, the risk aversion parameters are not obtained directly from option prices as in (2). The Heston model is estimated by minimising the squared error between market implied volatility and model implied volatility ${ }^{2}$

${ }^{2}$ The required calculations for this method are computationally extensive and the weighting approach by [36] helps to reduce the amount of computations. 


\subsubsection{Expected Risk-Aversion}

In line with [11] [13] and [17], the risk-aversion coefficient can be determined by the relation of the utility function to the risk-neutral and objective density function. The absolute risk-aversion, RA, can be then defined as:

$$
R A(x)=-\frac{u^{\prime \prime}(x)}{u^{\prime}(x)}=\frac{f_{P}^{\prime}(x)}{f_{P}(x)}-\frac{f_{Q}^{\prime}(x)}{f_{Q}(x)}
$$

Using this result, the relative risk-aversion, $\gamma$, can be retrieved by:

$$
\gamma=x R A(x) \text {. }
$$

Since it is assumed that the transformation from risk-neutral to subjective dynamics only impacts the mean return, the risk-aversion estimate is the same at every point $x$.

\section{Simulations}

This simulation exercise aims to unveil the influence of different characteristics of the underlying asset price process on risk aversion. As a starting point, we use the conventional Geometric Brownian Motion to model asset price dynamics and we then extend this to accommodate stochastic volatility, as discussed in Section 3. The simulations provide useful insights about how and to what extent risk-aversion estimates are influenced by different factors within the considered models. The main point of interest is the influence of stochastic volatility on the risk-aversion estimates.

Therefore, our simulations below include six different scenarios and examine the impact on risk aversion, as depicted in Panels A-F in the Table 1, Table 2, and Figures 1-3. Simulation A evaluates the change in the mean return, while Simulation B evaluates the impact of volatility, under a Geometric Brownian Motion. The remainder panels focus on the presence of stochastic volatility. The volatility process is modelled by an Ornstein-Uhlenbeck process. Simulation C investigates on the effect of the mean reversion $\kappa$ of volatility. Simulation D evaluates the impact of fat-tailed distributions via the volatility of volatility $\xi$. Lastly, Simulation E and F evaluate the impact of skewness in conjunction with a fat-tailed distribution. The latter might be the empirically most relevant case which can provide guidance for practical purposes.

Each simulation scheme derives risk aversion over an estimation horizon of $H=120$ monthly periods. As described in Section 3, the risk-neutral and subjective parameters are kept constant over the entire estimation horizon to ensure only the current market dynamics are considered. The simulated option prices are obtained one month prior to expiry. One month consists of 21 trading days, which results in 252 trading days per year. Each day is discretised by ten sub-steps to assure a sufficient precision of the simulated asset prices. The entire simulation is repeated $N=5000$ times.

\subsection{Geometric Brownian Motion}

Within the Geometric Brownian Motion, both the mean, $\mu$, and the variance, $\sigma^{2}$, influence the mean and variation of the simulated risk-aversion estimates, 
Table 1. The table provides the results for all 6 simulations. The expected value of gamma $E[\gamma]$ is obtained from the theoretical result in section 3 . The average, median and mean squared error for all density types are the results of the simulation. The bold values for the mean squared error mark the lowest values in each row.

\begin{tabular}{|c|c|c|c|c|c|c|c|c|c|c|}
\hline \multirow[b]{2}{*}{$E[\gamma]$} & \multicolumn{3}{|c|}{ median } & \multicolumn{3}{|c|}{ average } & \multicolumn{3}{|c|}{ MSE } & \multirow[b]{2}{*}{ simulation } \\
\hline & $\log$ & mix-log & GB2 & $\log$ & mix-log & GB2 & $\log$ & mix-log & GB2 & \\
\hline 0.00 & 0.00 & 0.07 & 0.02 & 0.01 & 0.03 & 0.03 & 10.17 & 8.48 & 9.58 & \multirow{5}{*}{ A } \\
\hline 1.50 & 1.50 & 1.25 & 1.47 & 1.48 & 1.43 & 1.46 & 9.73 & 7.11 & 9.18 & \\
\hline 2.50 & 2.60 & 2.59 & 2.54 & 2.59 & 2.58 & 2.53 & 9.91 & 9.78 & 9.29 & \\
\hline 3.50 & 3.46 & 3.42 & 3.36 & 3.49 & 3.45 & 3.40 & 10.18 & 9.91 & 9.50 & \\
\hline 4.50 & 4.51 & 3.83 & 4.38 & 4.48 & 4.15 & 4.37 & 9.88 & 6.10 & 9.22 & \\
\hline 0.00 & 0.08 & 0.13 & 0.09 & 0.09 & 0.11 & 0.11 & 9.86 & 8.74 & 9.27 & \multirow{4}{*}{ B } \\
\hline 0.33 & 0.35 & 0.35 & 0.35 & 0.37 & 0.37 & 0.38 & 3.40 & 3.39 & 3.21 & \\
\hline 0.40 & 0.37 & 0.37 & 0.38 & 0.38 & 0.38 & 0.38 & 1.93 & 1.93 & 1.84 & \\
\hline 0.44 & 0.42 & 0.42 & 0.43 & 0.42 & 0.42 & 0.42 & 1.13 & 1.13 & 1.07 & \\
\hline 0.00 & -0.08 & 0.04 & -0.11 & -0.05 & 0.03 & -0.10 & 41.77 & 26.22 & 29.63 & \multirow{5}{*}{$C$} \\
\hline 0.00 & -0.12 & -0.01 & -0.03 & -0.15 & -0.05 & 0.03 & 20.95 & 14.07 & 16.58 & \\
\hline 0.00 & -0.06 & 0.00 & -0.01 & -0.07 & -0.01 & -0.04 & 17.23 & 13.73 & 12.83 & \\
\hline 0.00 & -0.05 & 0.01 & 0.00 & -0.10 & -0.04 & 0.02 & 14.53 & 11.44 & 11.21 & \\
\hline 0.00 & -0.05 & 0.00 & -0.05 & -0.06 & -0.01 & -0.03 & 13.24 & 11.01 & 10.92 & \\
\hline 0.00 & 0.03 & 0.05 & 0.11 & -0.01 & 0.01 & 0.12 & 10.12 & 9.23 & 9.48 & \multirow{4}{*}{$\mathrm{D}$} \\
\hline 0.00 & 0.02 & 0.03 & 0.04 & 0.02 & 0.03 & -0.01 & 11.38 & 10.74 & 10.31 & \\
\hline 0.00 & -0.07 & 0.11 & 0.05 & -0.05 & 0.01 & -0.03 & 15.75 & 9.85 & 13.58 & \\
\hline 0.00 & -0.13 & -0.03 & 0.00 & -0.14 & -0.06 & 0.01 & 43.43 & 30.26 & 30.43 & \\
\hline 0.00 & -0.07 & 0.14 & -0.04 & -0.05 & -0.02 & -0.04 & 9.97 & 7.17 & 9.22 & \multirow{5}{*}{$\mathrm{E}$} \\
\hline 0.00 & -0.05 & -0.03 & -0.02 & 0.05 & 0.05 & 0.06 & 9.82 & 9.61 & 9.11 & \\
\hline 0.00 & 0.01 & 0.01 & 0.05 & 0.17 & 0.17 & 0.18 & 10.62 & 10.51 & 9.79 & \\
\hline 0.00 & 0.04 & 0.08 & 0.08 & 0.21 & 0.22 & 0.22 & 10.11 & 9.31 & 9.29 & \\
\hline 0.00 & -0.04 & -0.01 & 0.02 & 0.19 & 0.20 & 0.20 & 10.21 & 9.67 & 9.24 & \\
\hline 0.00 & -0.04 & 0.13 & 0.15 & 0.15 & 0.20 & 0.19 & 43.50 & 29.65 & 30.79 & \multirow{5}{*}{$F$} \\
\hline 0.00 & -0.02 & 0.22 & 0.29 & 0.36 & 0.38 & 0.38 & 42.20 & 28.61 & 30.06 & \\
\hline 0.00 & -0.30 & 0.10 & 0.17 & 0.46 & 0.46 & 0.46 & 45.14 & 28.50 & 30.14 & \\
\hline 0.00 & -0.02 & 0.41 & 0.53 & 0.82 & 0.77 & 0.78 & 45.08 & 27.45 & 29.34 & \\
\hline 0.00 & -0.27 & 0.31 & 0.45 & 0.73 & 0.69 & 0.70 & 48.53 & 27.89 & 30.13 & \\
\hline
\end{tabular}

respectively. Figures 1-3 A displays the case of an stepwise increase of $\mu$ from $-0.5 \%$ to $4 \%$. It is apparent that risk-aversion increases when the expected return rises since the compensation for risk becomes higher. Consequently, the mean of the subjective density shifts to reflect the premium in the underlying asset price process. 
Table 2. The table contains the average maximum log-likelihood estimate based on Equation (4) for the risk-neutral density (RND) and subjective density (RWD). The bold values mark the highest average value of log-likelihood in each row.

\begin{tabular}{|c|c|c|c|c|c|c|}
\hline \multicolumn{2}{|c|}{$\log$} & \multicolumn{2}{|c|}{ mix-log } & \multicolumn{2}{|c|}{ GB2 } & \multirow[b]{2}{*}{ simulation } \\
\hline RND & RWD & RND & RWD & RND & RWD & \\
\hline 0.57 & 1.08 & 0.48 & 0.96 & 0.00 & 0.49 & \\
\hline 0.47 & 1.07 & 0.42 & 0.96 & 0.00 & 0.58 & \\
\hline 0.46 & 1.29 & 0.46 & 1.28 & 0.00 & 0.81 & $A$ \\
\hline 0.45 & 1.56 & 0.40 & 1.50 & 0.00 & 1.08 & \\
\hline 0.43 & 1.94 & 0.37 & 1.72 & 0.00 & 1.47 & \\
\hline 0.45 & 0.95 & 0.34 & 0.81 & 0.00 & 0.48 & \\
\hline 0.50 & 1.03 & 0.50 & 1.03 & 0.00 & 0.52 & \\
\hline 0.42 & 0.94 & 0.42 & 0.94 & 0.00 & 0.51 & $B$ \\
\hline 0.43 & 1.02 & 0.43 & 1.02 & 0.00 & 0.57 & \\
\hline 0.46 & 1.05 & 0.46 & 1.05 & 0.00 & 0.58 & \\
\hline 0.00 & 1.81 & 111.32 & 112.80 & 119.66 & 121.21 & \\
\hline 0.00 & 0.92 & 42.47 & 43.23 & 43.48 & 44.31 & \\
\hline 0.00 & 0.77 & 24.70 & 25.39 & 28.18 & 28.83 & $C$ \\
\hline 0.00 & 0.66 & 19.04 & 19.62 & 18.81 & 19.38 & \\
\hline 0.00 & 0.61 & 13.26 & 13.81 & 14.93 & 15.48 & \\
\hline 0.00 & 0.50 & 0.48 & 0.97 & 1.97 & 2.45 & \\
\hline 0.00 & 0.56 & 8.84 & 9.38 & 10.75 & 11.27 & \\
\hline 0.00 & 0.75 & 22.30 & 22.92 & 29.28 & 29.97 & $\mathrm{D}$ \\
\hline 0.00 & 1.20 & 56.31 & 57.39 & 68.88 & 69.96 & \\
\hline 0.00 & 1.88 & 102.91 & 104.52 & 124.70 & 126.30 & J \\
\hline 0.00 & 0.50 & 0.27 & 0.70 & 2.14 & 2.61 & \\
\hline 0.00 & 0.49 & 0.81 & 1.30 & 2.18 & 2.65 & \\
\hline 0.00 & 0.53 & 0.52 & 1.04 & 2.21 & 2.72 & $\mathrm{E}$ \\
\hline 0.00 & 0.50 & 0.74 & 1.22 & 2.23 & 2.72 & \\
\hline 0.00 & 0.51 & 0.98 & 1.48 & 2.38 & 2.86 & \\
\hline 0.00 & 1.88 & 99.49 & 101.07 & 119.19 & 120.82 & \\
\hline 0.00 & 1.81 & 95.83 & 97.34 & 117.18 & 118.73 & \\
\hline 0.00 & 1.91 & 104.51 & 106.06 & 117.58 & 119.18 & $\mathrm{~F}$ \\
\hline 0.00 & 1.89 & 99.33 & 100.82 & 112.75 & 114.29 & \\
\hline 0.00 & 2.00 & 104.96 & 106.50 & 113.51 & 115.09 & \\
\hline
\end{tabular}

Figures 1-3 B displays the results of a change in variance. Compared to the previous case an increase in the variance does not result in a major increase of risk-aversion. The variance mainly influences the variation of risk-aversion. However, this relation is inverse, meaning when variance is high (low) the variation of risk aversion is low (high). This feature can be confirmed by (7), which clearly illustrates that the risk-premium is linked to the product of 


\section{LN}
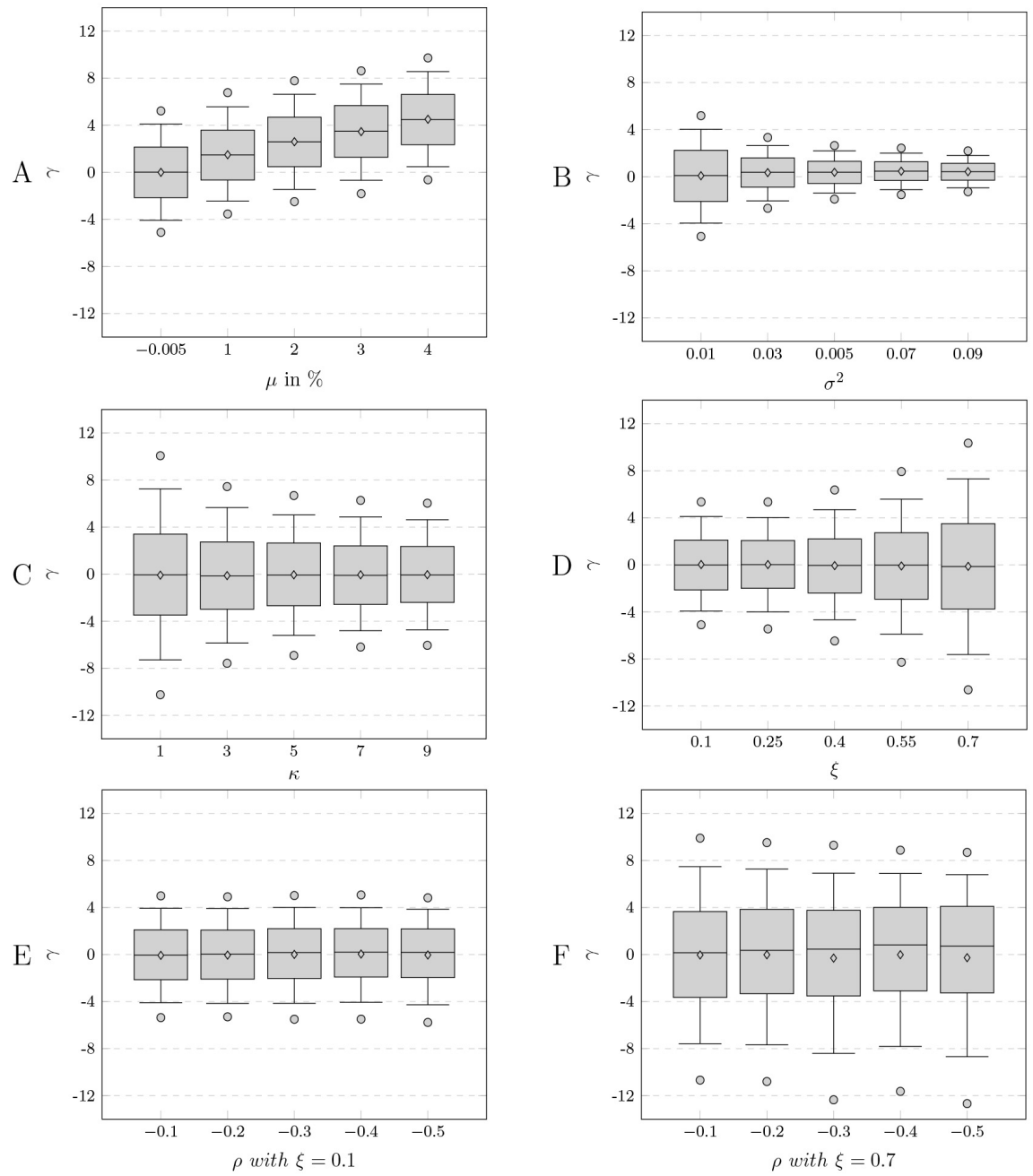

Figure 1. Distribution of estimated $\gamma$ s applying the lognormal density. A and B assume geometric Brownian motion as underlying process. The remaining plots are simulated applying the stochastic volatility model. The body of box plot is defined by the interquartile range of $\gamma$ estimates. The horizontal line dividing the box and diamond mark the median and average, respectively. The "whiskers" define the $10^{\text {th }}$ and $90^{\text {th }}$ percentile and the dots the $5^{\text {th }}$ and $95^{\text {th }}$ percentile. If not separately specified in the graph the parameter values for $\mu=-0.005, r=0, q=0$ and $\sigma^{2}=0.01$. For the case of the stochastic volatility model $\kappa=1, \theta=0.01, \xi=0.7$ and $\rho=0$.

volatility and risk-aversion. Thus, an increase in volatility results in lower variation of risk-aversion since the risk-aversion has to change less to achieve a specific premium

For both simulations, the mean squared error behaves similar for all considered density types but results change across specifications. In simulation A, the mean squared error is similar independently of the values for $\mu$. In contrast, the mean squared error varies heavily in simulation $B$ due to the changes in variance. In both cases mean and median infer that risk aversion is distributed symmetrically. 


\section{MLN}
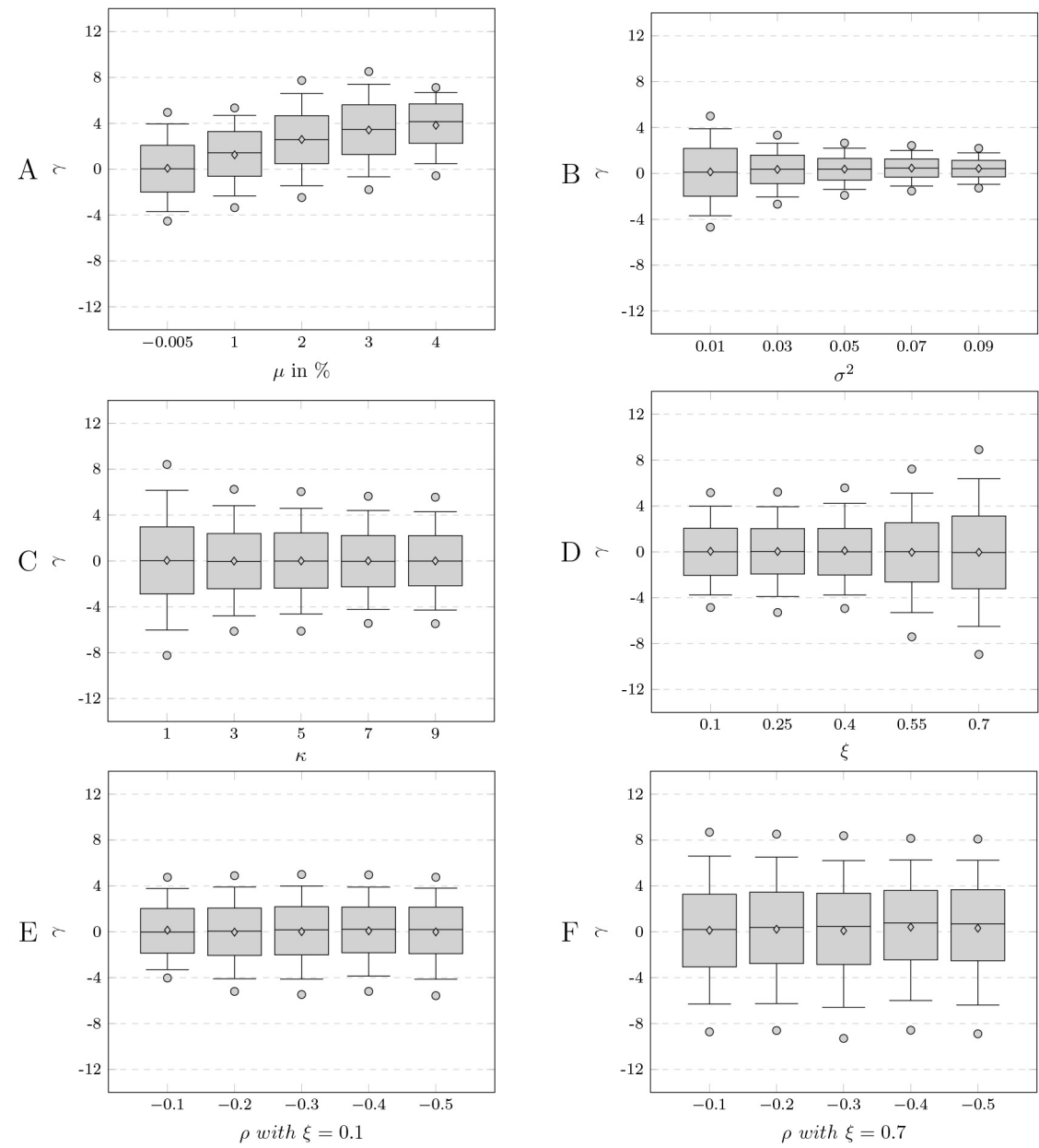

Figure 2. Distribution of estimated $\gamma$ s applying the mixture-lognormal density. A and B assume geometric Brownian motion as underlying process. The remaining plots are simulated applying the stochastic volatility model. The body of box plot is defined by the interquartile range of $\gamma$ estimates. The horizontal line dividing the box and diamond mark the median and average, respectively. The "whiskers" define the $10^{\text {th }}$ and $90^{\text {th }}$ percentile and the dots the $5^{\text {th }}$ and $95^{\text {th }}$ percentile. If not separately specified in the graph the parameter values for $\mu=-0.005, r=0, q=0$ and $\sigma^{2}=0.01$. For the case of the stochastic volatility model $\kappa=1, \theta=0.01, \xi=0.7$ and $\rho=0$.

\subsection{Stochastic Volatility}

Extending the Geometric Brownian motion to accommodate stochastic volatility allows to further analyse the impact of volatility on the underlying asset. We focus on the effect of the following parameters: the speed of mean reversion to the long run volatility, $\kappa$, the volatility of volatility, $\xi$, and the correlation between the two Wiener processes of the stock price and volatility, $\rho$. Figures 1-3 C displays the results across different values of $\kappa$. The results suggest that an increase in $\kappa$ has a similar influence as an increase in variance which can straightforwardly be explained by the mechanics of $\kappa$. A high value of $\kappa$ results in a faster reversion to the long run mean of the variance. 


\section{GB2}
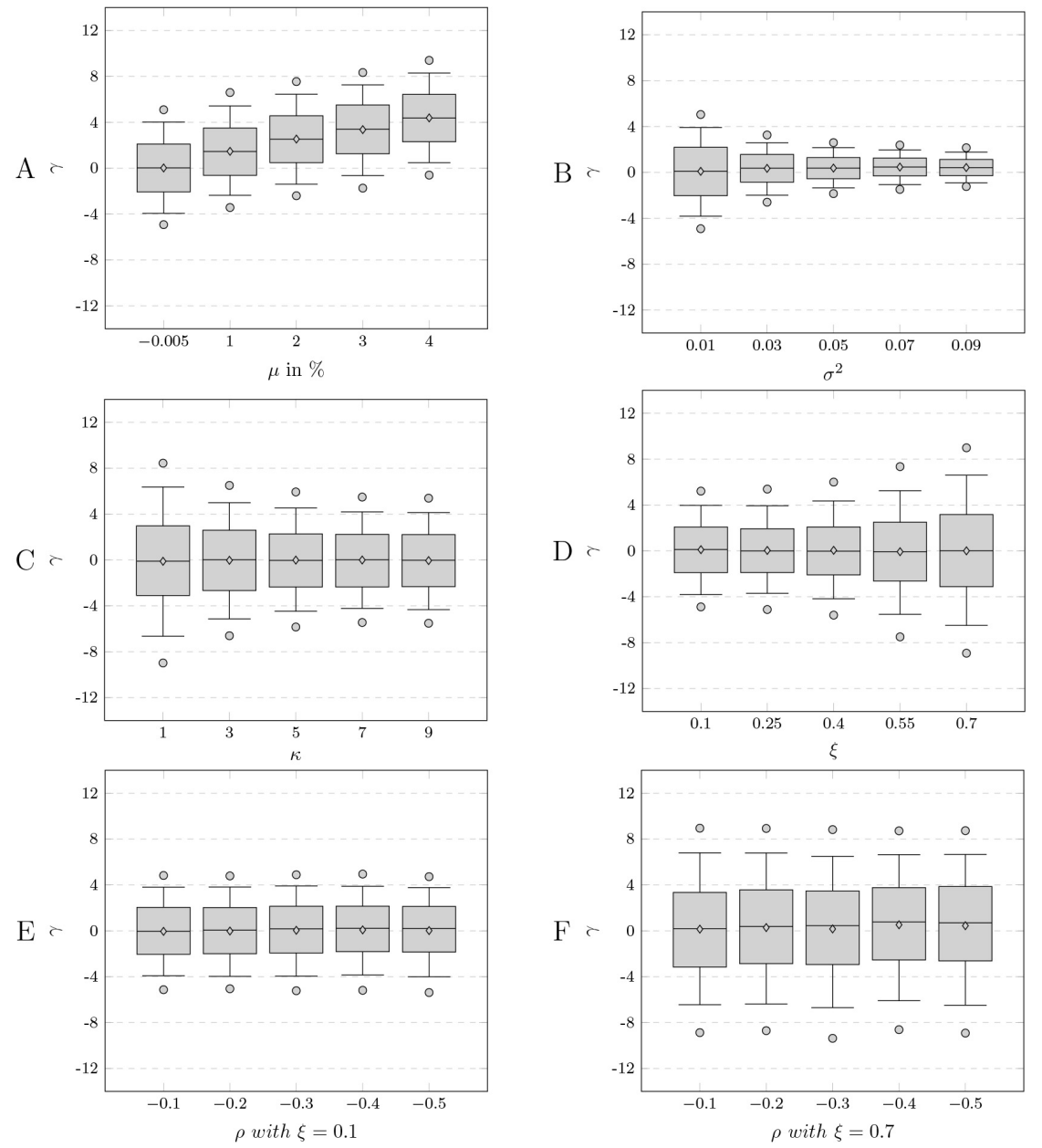

Figure 3. Distribution of estimated $\gamma$ s applying the GB2 density. A and B assume geometric Brownian motion as underlying process. The remaining plots are simulated applying the stochastic volatility model. The body of box plot is defined by the interquartile range of $\gamma$ estimates. The horizontal line dividing the box and diamond mark the median and average, respectively. The "whiskers" define the $10^{\text {th }}$ and $90^{\text {th }}$ percentile and the dots the $5^{\text {th }}$ and $95^{\text {th }}$ percentile. If not separately specified in the graph the parameter values for $\mu=-0.005, r=0, q=0$ and $\sigma^{2}=0.01$. For the case of the stochastic volatility model $\kappa=1, \theta=0.01, \xi=0.7$ and $\rho=0$.

In contrast, the parameter $\xi$ has an opposite effect on risk aversion, as shown in Figure 1-3 D. Increasing $\xi$ results in more variation of risk-aversion. This can be attributed to the fact that $\xi$ models the tail thickness of the asset price distribution. The simulation unveils that a higher frequency of extreme outcomes outweighs the impact of higher volatility, resulting in higher variation of risk-aversion.

Simulations $\mathrm{E}$ and $\mathrm{F}$ elaborate on the remaining parameter $\rho$, which captures asymmetry in the asset price distribution. Figures 1-3 E and F exhibit the influence of $\rho$ on the risk aversion and its interaction with $\xi$. Figures 1-3 $\mathrm{E}$ assumes a low value of $\xi$, whereas in Figures $1-3 \mathrm{~F} \xi$ is relatively high. 
Both plots show that with an increase in $\rho$ the asymmetry of the relative risk-aversion coefficient increases. This can be seen by the diverging mean and median parameters but also in the shrinking upper percentiles. The higher $\xi$ is the greater the absolute difference between median and average is. The influence of $\rho$ on the mean squared error is only minor as this is primarily driven by $\xi$.

In addition to Mean Square Error consideration, we also provide simulation results on the maximum likelihood estimates from the transformation from risk-neutral to subjective density and compare them to the risk-neutral likelihoods. The results are tabulated in Table 2 and draw a more complete picture along with results on mean squared error. In simulation $\mathrm{A}$ and $\mathrm{B}$ values are fairly close to each other. However, this changes from simulation $\mathrm{C}$ and ongoing. It shows that the GB2 density clearly fits the data the best, enforcing that a flexible density type is preferable when the underlying asset price distribution moves further away from lognormal. Furthermore, when the subjective density is compared to the risk-neutral density the likelihood is in all cases higher suggesting an improved fit.

Collectively, simulation results indicate that the main influence on the certainty of risk-aversion may be attributed to the volatility and the volatility of volatility. The mean mainly shifts the risk-aversion estimate in one or the other direction. In addition, flexible class of densities enable to obtain more accurate estimates for risk-aversion when the asset price distribution is skewed and has excess kurtosis. The fact that more flexible density types are favourable could also be shown by the likelihood comparison of the simulation. Finally, variation of risk-aversion strongly depends on the volatility component. Beyond that it could be shown that asymmetry in the asset price distribution also skews risk-aversion estimates. All these results provide useful insights to practitioners and highlight the importance of accounting for volatility when evaluating risk aversion estimates.

\section{Empirical Application}

\subsection{Data and Estimation}

This study considers European call and put options, which can only be exercised at the maturity date. We obtain option prices from Optionmetrics for the period from January 1996 until May 2016 on the S\&P 500 index. The considered expiry dates are 28 days before the third Friday of each month. We only consider options 28 days before expiry to ensure that estimation horizons are non-overlapping. In case that there are no option prices available for the 28 days before expiry, the observation date is shifted by one day. If there are still no options available the observation date is dropped from the sample. This results in 242 observation dates for the time period under study. The relevant option prices are selected based on their moneyness. All call (put) options with a moneyness greater (smaller) than $1.03(0.97)$ are removed from the sample since they are less frequently traded. Moneyness is defined as the ratio between strike price and 
underlying stock price. Furthermore, if there is no traded volume the option is removed as well. The estimation of the risk-neutral density is then performed taking into account the mid option price. Put options are transformed into call-options by the put-call parity relationship. In case there are two options available for the same strike price, the average mid price is used. In case dividends and zero-coupon rates do not match the maturity date, rates are interpolated between the two closest dates. Future prices $F$ are calculated synthetically by the pricing equation $F_{t}=S_{t} \mathrm{e}^{\left(r_{t}-q_{t}\right) T}$ (Table 3).

The risk-neutral density parameters are estimated minimising (2) at each observation date. Multiple start values were supplied into the minimisation problem to ensure the minimum is robust. Table 4 provides the summary statistics of the option pricing errors. The reported values are in a similar range as, for example, [24]. There are remarkable differences between the more flexible densities and the lognormal approach. The pricing errors can attributed to the greater flexibility of the MLN and GB2, which better replicate the non-normal distribution contained in option prices.

\subsection{Results}

The empirical results, applying the simulation method to observable S\&P 500 index options, are presented in Figure 4 and Figure 5. The graphs display the mean and median simulated risk-aversion estimate enclosed by confidence intervals. Furthermore, the estimation using a sequence of risk-neutral densities and asset price outcomes as in [1] is displayed. Their procedure is denoted as "historical approach".

For the case of the Geometric Brownian Motion, the results of the different density types are similar with no major discrepancies. Overall, the simulation method suggests a symmetric distribution of risk aversion since the mean and median are close to each other and the percentiles are evenly distributed. Furthermore, during times of high volatility, as for example at the subprime crisis, the variation in risk aversion lowers. These observations confirm the results from the simulation.

More complex are the findings assuming stochastic volatility for the price process. The greater flexibility of the underlying process allows for an asymmetric shape. While in the previous case the mean and median for all density types are close together, they diverge for the lognormal case. The median tends to be in excess towards the median, imposing a negative skew. This observation is a result of the negative skew induced by the stochastic volatility process. The lognormal distribution is not able to accommodate the more complex shape of the underlying probability distribution. Therefore, the estimation of the risk-aversion parameter compensates for this inflexibility, resulting in a skewed distribution of risk aversion. This observation highlights the importance of flexible density types when trying to estimate risk aversion. This finding lines up with the characteristics of the simulation, that more flexible density types yield a lower mean square error. Furthermore, the confidence 
Table 3. Total number of options and options by moneyness.

(a)

\begin{tabular}{ccccc}
\hline & Total number & Average & Max & Min \\
\hline Calls & 7411 & 31 & 104 & 7 \\
Puts & 12,857 & 53 & 264 & 11 \\
Overall & 20,268 & 84 & 350 & 20 \\
\hline
\end{tabular}

\begin{tabular}{cccc}
\hline \multicolumn{3}{c}{ (b) } \\
\hline Moneyness & $\mathrm{S} / \mathrm{K}$ & Total number & In \% \\
\hline Deep OTM put & $>1.10$ & 6629 & 32.71 \\
OTM put & $1.03-1.10$ & 3106 & 15.32 \\
Near the money & $0.97-1.03$ & 6342 & 31.29 \\
OTM call & $0.90-0.97$ & 3159 & 15.59 \\
Deep OTM call & $<0.90$ & 1032 & 5.09 \\
Overall & & 20,268 & 100.00 \\
\hline
\end{tabular}

Table 4. This table provides the summary statistics when estimating the risk-neutral density. The estimation is performed as in 2 and minimises the squared error between model and observed option prices.

\begin{tabular}{cccc}
\hline Density & Median & Average & Standard deviation \\
\hline LN & 3.21 & 4.89 & 6.04 \\
MLN & 0.10 & 0.16 & 0.17 \\
GB2 & 0.09 & 0.17 & 0.22 \\
\hline
\end{tabular}

intervals have a stronger variation compared to the Geometric Brownian Motion. This is not a surprise due to the greater complexity of stochastic volatility. However, remarkable are the differences in the lower confidence intervals. For the MLN and GB2, they swing far less into negative values of risk aversion, which further highlights the importance of flexible density types.

Overall, it is remarkable how uncertain the estimates are even within basic assumptions as displayed in Figure 4 and Figure 5. Generally, the simulationbased methods tend to exceed the risk-aversion estimates of the historical approach. Nevertheless, the confidence intervals mostly encompass the historical estimates, particularly when assuming stochastic volatility.

To gain further insight, we use the obtained mean risk-aversion estimate and transform the risk-neutral density into its subjective counterpart. Afterwards, we evaluate their predictability based on the log-likelihood of the final asset price outcomes. This results in 122 evaluation periods since the historical method retrieves the estimates using a 10 year rolling window. The log-likelihood is reported in Table 5 in excess to the risk-neutral LN. The reported values can clearly show that the more flexible density types are preferable towards the LN. Furthermore, the GB2 is clearly in excess towards the MLN. The additional 


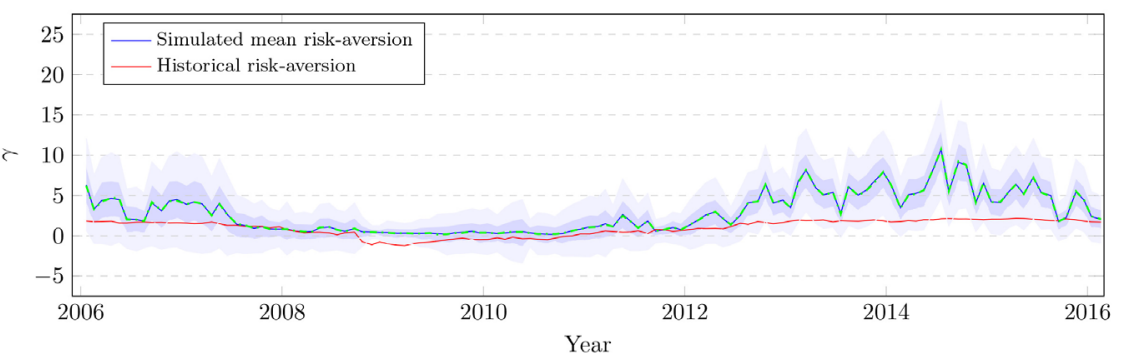

MLN

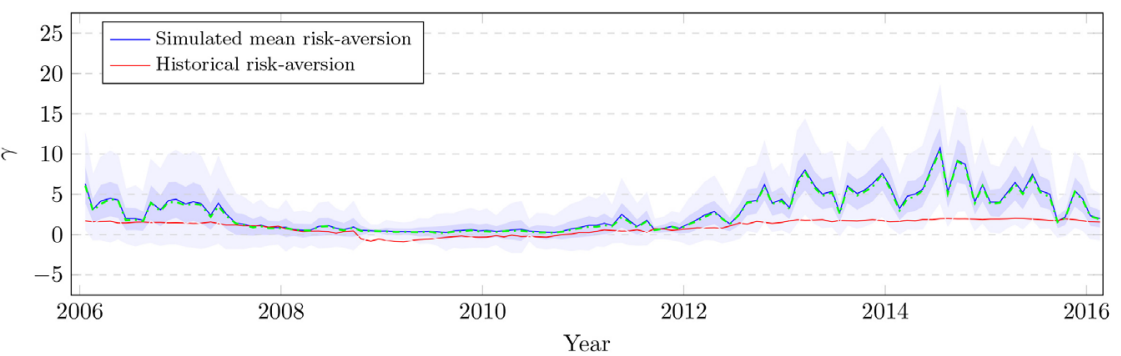

GB2

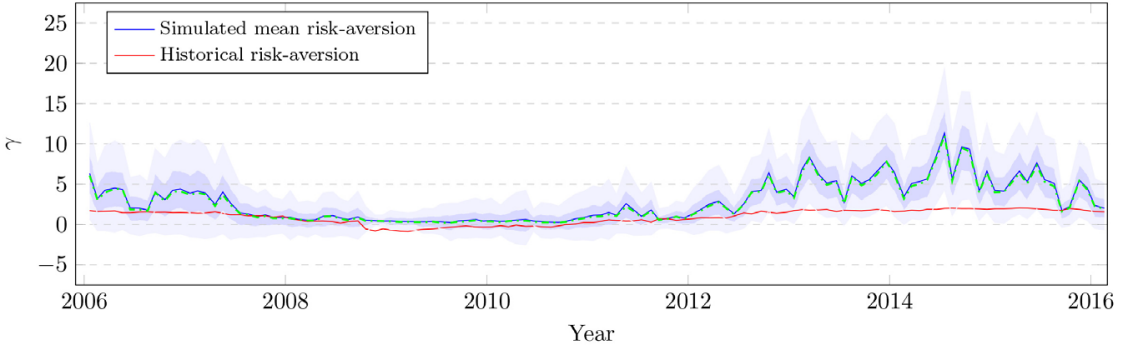

Figure 4. Risk-aversion simulation results and historical estimates. The simulation assumes the asset price follows a geometric Brownian motion. The dark blue line denotes the average estimate of risk aversion whereas the green dashed-dotted line denotes the median. The inner confidence interval (dark blue) defined by the 25 th and $75_{\text {th }}$ percentile. The outer confidence interval (light blue) is defined by the $5^{\text {th }}$ and $95^{\text {th }}$ percentile. The red line defines the risk-aversion obtained by the classic method.

parameter $\gamma$ is further tested using the log-likelihood ratio test. All methods are significant at the $20 \%$ level. The reason for the low significance level could be rooted in the short sample size. [27] encounter similar difficulties and argue the insignificance is a type II error. These authors base this claim on the findings of [37] and others since the risk-premium is small relative to volatility and, therefore, difficult to estimate.

Finally, we test two competing forecasts for equal expected log-likelihoods following [33]. The results are reported in Table 6. Comparing the different density types with each other clearly shows that the GB2 is the preferable method. The test rejects the null hypothesis at the $1 \%$ level in all cases. Within each density type findings are less clear. Only for the historical risk aversion, the subjective density is significantly different from the risk-neutral density. In none 
LN

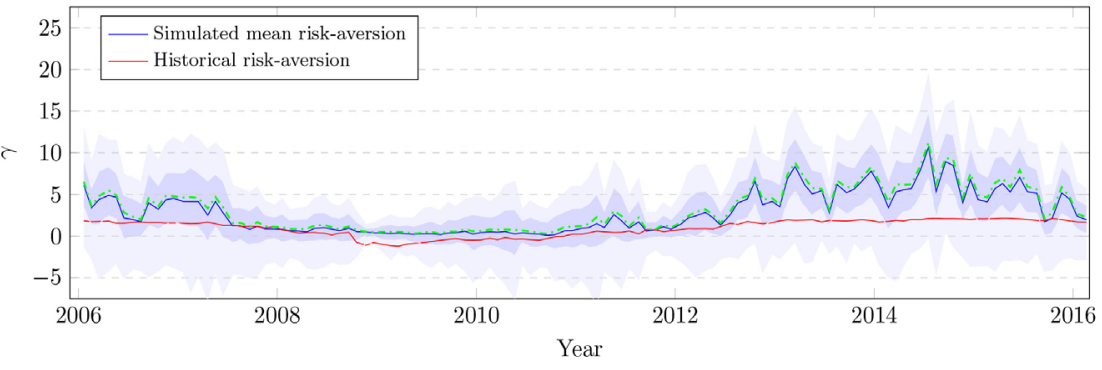

MLN

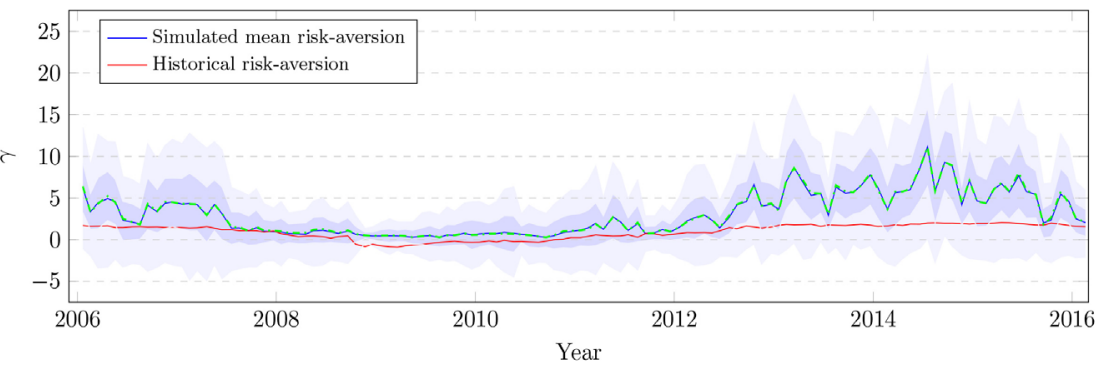

GB2

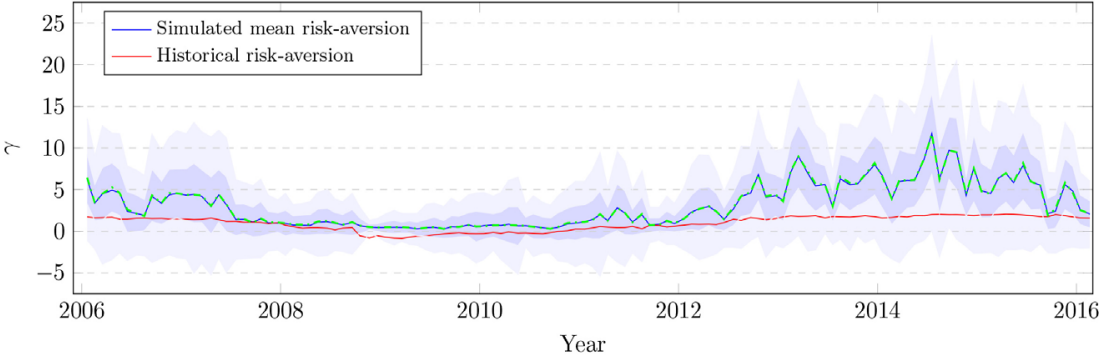

Figure 5. Risk-aversion simulation results and historical estimates. The simulation assumes the asset price follows stochastic volatility. The dark blue line denotes the average estimate of risk-aversion whereas the green dashed-dotted line denotes the median. The inner condence interval (dark blue) de ned by the $25^{\text {th }}$ and $75^{\text {th }}$ percentile. The outer con dence interval (light blue) is de ned by the $5^{\text {th }}$ and $95^{\text {th }}$ percentile. The red line de nes the risk-aversion obtained by the classic method.

Table 5. The excess log-likelihood is defined as excess value compared to the weakest method. In this case the worst performing method is the risk-neutral LN. The remaining methods denote transformed subjective densities.

\begin{tabular}{cccccccc}
\hline & \multicolumn{3}{c}{$\begin{array}{c}\text { Excess } \\
\text { log-likelihood }\end{array}$} & & \multicolumn{3}{c}{$2^{\star}$ LR } \\
\cline { 2 - 4 } \cline { 6 - 7 } Method & LN & MLN & GB2 & & LN & MLN & GB2 \\
\hline RND & 0.00 & 17.04 & 96.44 & & & \\
Hist. & 1.04 & 18.03 & 97.43 & & 2.09 & 1.69 & 1.98 \\
GBM & 1.20 & 18.17 & 97.58 & & 2.40 & 2.26 & 2.28 \\
SV & 1.18 & 18.17 & 97.58 & & 2.36 & 2.25 & 2.27 \\
\hline
\end{tabular}


Table 6. The table contains the test-statistics of the [33] test. The critical values are denoted with $\left({ }^{*}\right)$ at the $10 \%$ level, $\left({ }^{*}\right)$ at the $5 \%$ level and $(* * *)$ at the $1 \%$ level.

\begin{tabular}{|c|c|c|c|c|c|c|c|c|c|c|c|c|c|}
\hline & & \multicolumn{4}{|c|}{$\log$} & \multicolumn{4}{|c|}{$\operatorname{mix} \log$} & \multicolumn{4}{|c|}{ GB2 } \\
\hline & & RND & hist. & $\operatorname{sim} .1$ & $\operatorname{sim} .2$ & RND & hist. & $\operatorname{sim} .1$ & $\operatorname{sim} .2$ & RND & hist. & sim. 1 & $\operatorname{sim} .2$ \\
\hline \multirow{4}{*}{$\log$} & RND & & & & & & & & & & & & \\
\hline & hist. & $1.91^{*}$ & & & & & & & & & & & \\
\hline & $\operatorname{sim} .1$ & 1.13 & 0.18 & & & & & & & & & & \\
\hline & $\operatorname{sim} .2$ & 1.12 & 0.16 & 0.31 & & & & & & & & & \\
\hline \multirow{4}{*}{ mix log } & RND & $2.09^{* *}$ & $2.01^{\star \star}$ & $1.91^{*}$ & $1.91^{\star}$ & & & & & & & & \\
\hline & hist. & $2.16^{* *}$ & $2.09^{* *}$ & $2^{* *}$ & $2^{* *}$ & $2.08^{\star *}$ & & & & & & & \\
\hline & $\operatorname{sim} .1$ & $2.23^{\star \star}$ & $2.16^{* *}$ & $2.08^{\star *}$ & $2.08^{\star *}$ & 1.07 & 0.18 & & & & & & \\
\hline & $\operatorname{sim} .2$ & $2.23^{\star *}$ & $2.17^{\star *}$ & $2.09^{* *}$ & $2.09^{* *}$ & 1 & 0.16 & 0.06 & & & & & \\
\hline \multirow{4}{*}{ GB2 } & RND & $11.58^{\star * *}$ & $11.76^{\star * *}$ & $11.26^{\star * *}$ & $11.27^{\star * *}$ & $30.92^{* * *}$ & $30.71^{\star * *}$ & $28.25^{\star * \star}$ & $27.89^{\star * *}$ & & & & \\
\hline & hist. & $11.44^{* * *}$ & $11.65^{* * *}$ & $11.19^{* * *}$ & $11.19^{* * *}$ & $30.15^{* * *}$ & $30.93^{* * *}$ & $28.86^{* * *}$ & $28.55^{\star * *}$ & $2.11^{\star *}$ & & & \\
\hline & $\operatorname{sim} .1$ & $11.73^{* * *}$ & $11.97^{\star * *}$ & $11.59^{\star * *}$ & $11.59^{* * *}$ & $28.82^{* * *}$ & $29.91^{* * *}$ & $30.89^{* * *}$ & $30.79^{* * *}$ & 1.05 & 0.17 & & \\
\hline & sim. 2 & $11.74^{* * *}$ & $11.99^{* * *}$ & $11.62^{\star * *}$ & $11.62^{\star * *}$ & $28.58^{* * *}$ & $29.69^{* * *}$ & $30.94^{\star * *}$ & $30.88^{* * *}$ & 0.98 & 0.15 & 0.04 & \\
\hline
\end{tabular}

of the cases, the proposed simulation method is significantly different to the risk-neutral density. A possible explanation for this observation could be that the volatility dynamics are assumed to be the same in the risk-neutral and subjective model dynamics. It is well known that option prices contain a volatility premium and the volatility in the subjective process tends to be lower. Therefore, when assuming the risk-neutral and subjective volatility dynamics to be the same, we implicitly assume similar expected log-likelihoods (Table 6).

\section{Conclusions}

This paper provides a novel methodology for uncovering the presence of risk aversion in asset prices. Risk aversion is interpreted as the coefficient of relative risk aversion characterizing a power utility function. In contrast to most of the literature, we extract risk aversion from the risk-neutral distribution underlying the risk-neutral valuation of option prices. More specifically, we exploit a parametric expression relating the risk-neutral distribution function with an objective distribution describing the true probability distribution of asset prices and a power utility function describing individuals' risk attitude. This expression provides a closed-form solution that links risk-neutral expectations with a subjective valuation that incorporates the preferences of a representative agent. This approach allows us to estimate consistently the coefficient of relative risk aversion as the sample size increases.

We have also proposed a simulation scheme to introduce uncertainty into the pricing models. By doing so, we have constructed confidence intervals that 
reflect the uncertainty around the parameter estimates of relative risk aversion. We have also accommodated the presence of dynamics in risk aversion by proposing a parametric model for describing the dynamics of asset prices. Finally, as an additional contribution, we have explored different types of density functions for describing the probability law of asset prices. By doing so, we have explored the predictive ability of the model and assessed, out of sample, the reliability of the different estimates of the relative risk-aversion coefficient for prediction purposes.

Our results, obtained from an extensive simulation exercise, suggest that there is more variation in the risk-aversion coefficient than implied by [1]. Not surprisingly, the volatility component has a major impact on the coefficient of relative risk aversion. This is due to the direct link between volatility, risk aversion and the risk premium. A way to make the risk-aversion estimate less varying and more stable across time is to assume more flexible density types. Especially, when stochastic volatility is present more flexible density types obtain more stable values. The choice of a parametric model for describing asset prices also has a major effect on the estimates of risk aversion. Thus, we note that imposing a lognormal density on asset prices produces greater variation in the coefficient of risk aversion compared to the mixture-lognormal and generalised beta distribution of the second kind. Lastly, we also perform a forecasting exercise to assess the relative predictive ability of each estimation method out of sample. For all density types, there is no statistically significant difference between the risk-aversion coefficient based on the Monte-Carlo simulation and the classic approach developed by [1]. In contrast, comparing both approaches against the risk-neutral density reveals statistically significant differences.

Within this paper we centred our attention on the classic case of a power utility function. Therefore, it can be extended in different directions to obtain further insights into the degree of risk aversion in financial markets and its variation over time. First, an interesting avenue for further research is to evaluate the influence of the volatility premium on risk-aversion estimates. Second, exploring alternative utility functions that provide a different characterization of individuals' risk aversion can be a fruitful strategy to uncover different patterns of risk aversion under different assumptions on individuals' risk profile. Finally, our simulation exercise clearly shows that individuals' risk aversion changes over time. It might be of much interest to applied economists and financial practitioners to explore suitable time series models for forecasting asset prices constructed from reliable estimates of the risk-aversion coefficient.

\section{Conflicts of Interest}

The authors declare no conflicts of interest regarding the publication of this paper.

\section{References}

[1] Bliss, R.R. and Panigirtzoglou, N. (2004) Option-Implied Risk Aversion Estimates. 
Journal of Finance, 59, 407-446. https://doi.org/10.1111/j.1540-6261.2004.00637.x

[2] Mehra, R. and Prescott, E.C. (1985) The Equity Premium: A Puzzle. Journal of Monetary Economics, 15, 145-161. https://doi.org/10.1016/0304-3932(85)90061-3

[3] Arrow, K.J. (1971) Essays in the Theory of Risk-Bearing. North Holland, Amsterdam.

[4] Friend, I. and Blume, M.E. (1975) The Demand for Risky Assets. The American Economic Review, 65, 900-922.

[5] Hansen, L.P. and Singleton, K.J. (1982) Generalized Instrumental Variables Estimation of Nonlinear Rational Expectations Models. Econometrica, 50, 1269-1286. https://doi.org/10.2307/1911873

[6] Epstein, L.G. and Zin, S.E. (1991) Substitution, Risk Aversion, and the Temporal Behavior of Consumption and Asset Returns: An Empirical Analysis. Journal of Political Economy, 99, 263-286. https://doi.org/10.1086/261750

[7] Ferson, W.E. and Constantinides, G. (1991) Habit Persistence and Durability in Aggregate Consumption: Empirical Tests. Journal of Financial Economics, 29, 199-240. https://doi.org/10.1016/0304-405X(91)90002-2

[8] Cochrane, J.H. and Hansen, L.P. (1992) Asset Pricing Explorations for Macroeconomics. Working Paper 4088, National Bureau of Economic Research.

[9] Jorion, P. and Giovannini, A. (1993) Time-Series Tests of a Non-Expected-Utility Model of Asset Pricing. European Economic Review, 37, 1083-1100. https://doi.org/10.1016/0014-2921(93)90110-V

[10] Normandin, M. and St-Amour, P. (1998) Substitution, Risk Aversion, Taste Shocks and Equity Premia. Journal of Applied Econometrics, 13, 265-281. https://doi.org/10.1002/(SICI)1099-1255(199805/06)13:3<265::AID-JAE475>3.0.CO ;2-Z

[11] Aït-Sahalia, Y. and Lo, A. (2000) Nonparametric Risk Management and Implied Risk Aversion. Journal of Econometrics, 94, 9-51. https://doi.org/10.1016/S0304-4076(99)00016-0

[12] Guo, H. and Whitelaw, R. (2001) Risk and Return: Some New Evidence. Working Paper No. 2001-001 A, Federal Reserve Bank of St. Louis.

[13] Jackwerth, J.C. (2000) Recovering Risk Aversion from Option Prices and Realized Returns. The Review of Financial Studies, 13, 433-451. https://doi.org/10.1093/rfs/13.2.433

[14] Aït-Sahalia, Y., Wang, Y.B. and Yared, F. (2001) Do Option Markets Correctly Price the Probabilities of Movement of the Underlying Asset? Journal of Econometrics, 102, 67-110. https://doi.org/10.1016/S0304-4076(00)00091-9

[15] Weinberg, S.A. (2001) Interpreting the Volatility Smile: An Examination of the Information Content of Option Prices. Discussion Paper, FRB International Finance.

[16] Prignon, C. and Villa, C. (2002) Extracting Information from Options Markets: Smiles, Stateprice Densities and Risk Aversion. European Financial Management, 8, 495-513. https://doi.org/10.1111/1468-036X.00201

[17] Rosenberg, J.V. and Engle, R.F. (2002) Empirical Pricing Kernels. Journal of Financial Economics, 64, 341-372. https://doi.org/10.1016/S0304-405X(02)00128-9

[18] Kliger, D. and Levy, O. (2002) Risk Preferences Heterogeneity: Evidence from Asset Markets. Review of Finance, 6, 277-290. https://doi.org/10.1023/A:1022069113893

[19] Anagnou-Basioudis, I., Bedendo, M., Hodges, S.D. and Tompkins, R. (2005) Forecasting Accuracy of Implied and Garch-Based Probability Density Functions. Review of Futures Markets, 11, 41-66. 
[20] Ziegler, A. (2007) Why Does Implied Risk Aversion Smile? The Review of Financial Studies, 20, 859-904. https://doi.org/10.1093/rfs/hhl023

[21] Bates, D.S. (2000) Post-'87 Crash Fears in the s\&p 500 Futures Option Market. Journal of Econometrics, 94, 181-238. https://doi.org/10.1016/S0304-4076(99)00021-4

[22] Chernov, M. and Ghysels, E. (2000) A Study towards a Unified Approach to the Joint Estimation of Objective and Risk Neutral Measures for the Purpose of Options Valuation. Journal of Financial Economics, 56, 407-458. https://doi.org/10.1016/S0304-405X(00)00046-5

[23] Pan, J. (2002) The Jump-Risk Premia Implicit in Options: Evidence from an Integrated Time-Series Study. Journal of Financial Economics, 63, 3-50. https://doi.org/10.1016/S0304-405X(01)00088-5

[24] Shackleton, M.B., Taylor, S.J. and Yu, P. (2010) A Multi-Horizon Comparison of Density Forecasts for the s\&p 500 Using Index Returns and Option Prices. Journal of Banking \& Finance, 34, 2678-2693. https://doi.org/10.1016/j.jbankfin.2010.05.006

[25] Fackler, P.L. and King, R.P. (1990) Calibration of Option-Based Probability Assessments in Agricultural Commodity Markets. American Journal of Agricultural Economics, 72, 73-83. https://doi.org/10.2307/1243146

[26] Kang, B.J. and Kim, T.S. (2006) Option-Implied Risk Preferences: An Extension to Wider Classes of Utility Functions. Journal of Financial Markets, 9, 180-198. https://doi.org/10.1016/j.finmar.2005.12.004

[27] Liu, X., Shackleton, M.B., Taylor, S.J. and Xu, X. (2007) Closed-Form Transformations from Risk-Neutral to Real-World Distributions. Journal of Banking \& Finance, 31, 1501-1520. https://doi.org/10.1016/j.jbankfin.2006.09.005

[28] Breeden, D.T. and Litzenberger, R.H. (1978) Prices of State-Contingent Claims Implicit in Option Prices. The Journal of Business, 51, 621-651. https://doi.org/10.1086/296025

[29] Ritchey, R.J. (1990) Call Option Valuation for Discrete Normal Mixtures. Journal of Financial Research, 13, 285-296. https://doi.org/10.1111/j.1475-6803.1990.tb00633.x

[30] Black, F. (1976) The Pricing of Commodity Contracts. Journal of Financial Economics, 3, 167-179. https://doi.org/10.1016/0304-405X(76)90024-6

[31] Bookstaber, R.M. and McDonald, J.B. (1987) A General Distribution for Describing Security Price Returns. Journal of Business, 60, 401-424. https://doi.org/10.1086/296404

[32] Bao, Y., Lee, T.-H. and Saltolu, B. (2007) Comparing Density Forecast Models. Journal of Fore-Casting, 26, 203-225. https://doi.org/10.1002/for.1023

[33] Amisano, G. and Giacomini, R. (2007) Comparing Density Forecasts via Weighted Likelihood Ratio Tests. Journal of Business \& Economic Statistics, 25, 177-190. https://doi.org/10.1198/073500106000000332

[34] Black, F. and Scholes, M. (1973) The Pricing of Options and Corporate Liabilities. Journal of Political Economy, 81, 637-654. https://doi.org/10.1086/260062

[35] Heston, S.L. (1993) A Closed-Form Solution for Options with Stochastic Volatility with Applications to Bond and Currency Options. The Review of Financial Studies, 6, 327-343. https://doi.org/10.1093/rfs/6.2.327

[36] Christofiersen, P., Heston, S. and Jacobs, K. (2009) The Shape and Term Structure of the Index Option Smirk: Why Multifactor Stochastic Volatility Models Work So Well. Management Science, 55, 1914-1932. https://doi.org/10.1287/mnsc.1090.1065

[37] Merton, R.C. (1980) On Estimating the Expected Return on the Market. Journal of Financial Economics, 8, 323-361. https://doi.org/10.1016/0304-405X(80)90007-0 